11

\title{
Исследование и оптимизация характеристик генерации сверхмощного виртода
}

\author{
(C) А.А. Бадарин, ${ }^{1,2}$ C.А. Куркин, ${ }^{1,2}$ Н.С. Фролов, ${ }^{1,2}$ A.О. Рак, ${ }^{3}$ А.Е. Храмов ${ }^{1,2}$ \\ ${ }^{1}$ Саратовский национальный исследовательский государственный университет им. Н.Г. Чернышевского, \\ 410012 Саратов, Россия \\ ${ }^{2}$ Саратовский государственный технический университет им. Ю.А. Гагарина, \\ 410054 Саратов, Россия \\ ${ }^{3}$ Белорусский государственный университет информатики и радиоэлектроники, \\ 220013 Минск, Беларусь \\ e-mail: Badarin.a.a@mail.ru
}

(Поступило в Редакцию 15 марта 2018 г. В окончательной редакции 2 октября 2018 г.)

Представлены результаты трехмерного численного моделирования перспективной схемы генератора на виртуальном катоде с внешней обратной связью (виртода), а также оптимизации характеристик его генерации. Исследовано влияние параметров пучка на выходные характеристики виртода. В частности, получена зависимость КПД от величины питающего напряжения и инжектируемого тока. Исследовано влияние предмодуляции пучка по скорости и разброса электронов по скоростям на характеристики генерации виртода. Выявлено, что введение предмодуляции приводит к резкому увеличению эффективности генерации вплоть до 13\% при оптимальных параметрах. Показана возможность устойчивой генерации в виртоде при относительно больших разбросах электронов по скоростям.

DOI: 10.21883/JTF.2019.03.47181.113-18

\section{Введение}

В настоящее время исследование систем с релятивистскими электронными потоками (РЭП) является актуальной и важной проблемой современной физики плазмы и высокомощной СВЧ-электроники, что обусловлено существенной значимостью РЭП в следующих областях применения: нагрев плазмы, генерация сверхмощного СВЧ-излучения, ускорение ионов и др. $[1,2]$. Многие процессы, происходящие в релятивистских электронных потоках, например развитие различных типов пучковоплазменных неустойчивостей, все еще слабо изучены. Одним из важнейших направлений исследований в вакуумной и плазменной электронике больших мощностей является изучение и оптимизация релятивистских генераторов на виртуальном катоде, которые с конца 1970-х годов привлекают большое внимание научного сообщества [1,3-16]. Приборы на виртуальном катоде (такие как виркатор, редитрон, виртод и др.) представляют особый класс СВЧ-генераторов и усилителей тормозного излучения, работа которых основана на формировании виртуального катода (ВК) в электронном потоке (обычно релятивистском) со сверхкритическим током [1,3,5,17-20]. Интерес к подобным генераторам обусловлен их преимуществами: очень высокая выходная мощность СВЧ-излучения, простота конструкции (в частности, виркаторы могут работать без внешнего фокусирующего магнитного поля), быстрый старт генерации, возможность простой настройки частоты и переключения режимов, низкие требования к качеству электронного потока $[1,6,8,21,22]$. Последнее свойство имеет большое значение при инжекции в систему ко- роткоимпульсного и низкокачественного пучка, формируемого взрывоэмиссионной электронной пушкой [23].

Тем не менее генераторы на виртуальном катоде имеют некоторые существенные недостатки, такие как нестабильность частоты генерации (для некоторых схем), низкий КПД, которые ограничивают их широкое применение [1]. Введение дополнительной электромагнитной обратной связи в таких генераторах уменьшает вышеупомянутые нежелательные эффекты [24-26]. Перспективной модификацией генераторов на ВК с электромагнитной обратной связью является двухзазорный виртод, который был исследован, в частности, в работах [25-27].

В настоящее время в области изучения виртодов остается еще ряд важных вопросов, требующих дальнейших исследований. В частности, не было исследовано влияние качества электронного потока и его предмодуляции на характеристики генерации виртода; не проведена оптимизация прибора с целью достижения максимальной эффективности генерации. Таким образом, настоящая работа является продолжением исследований двухзазорного виртода и посвящена изучению обозначенных выше вопросов.

\section{Исследуемая модель}

Исследование характеристик генерации виртода было проведено с помощью хорошо зарекомендовавшего себя метода „частица в ячейке“ (РІС-метода), реализованного в трех пространственных координатах [28]. Данный метод основан на самосогласованном численном решении уравнений Максвелла методом конечных разностей во 
временной области (FDTD) и релятивистских уравнений движения, описывающих поведение частиц. Метод FDTD базируется на дискретизации уравнений Максвелла, записанных в дифференциальной форме в декартовой системе координат. При этом сетки для полей $E$ и $H$ сдвигаются относительно друг друга на половину шага дискретизации по времени и каждой из пространственных координат. Данная сетка называется сеткой Йи. Конечно-разностные уравнения Максвелла позволяют определить поля $E$ и $H$ на заданном временном шаге на основе известных значений полей на предыдущем шаге.

Итак, сначала вычисляются электромагнитные поля в соответствии с уравнениями Максвелла, затем решаются уравнения движения для каждой крупной частицы. При этом временной и пространственный шаги выбираются в соответствии с условием Куранта-Фридрихса-Леви [28]. В свою очередь, движение частиц определяет плотность тока, требующуюся для вычисления электромагнитных полей в соответствии с уравнениями Максвелла на очередном временном шаге. Таким образом, обеспечивается закон сохранения тока и заряда. В совокупности данный подход позволяет обеспечить высокую степень достоверности и точности полученных результатов.

Перейдем к рассмотрению исследуемой схемы виртода (рис. 1) [25]. Модель содержит следующие основные конструктивные элементы: электронную пушку 1 в виде цилиндрического вакуумного диода со взрывоэмиссионным катодом 2, служащую для создания интенсивного сплошного электронного потока 3 с плотностью тока порядка $10^{6}-10^{7} \mathrm{~A} / \mathrm{cm}^{2}$, анодную сетку 4, двухзазорный резонатор 5 с окном связи 6 между зазорами, сетку (обычно фольгу) 9 в стенке между зазорами и вывод мощности 12 в виде волновода с рупорной антенной, подключенный ко второму зазору резонатора, плунжеры 10 и 11, позволяющие регулировать ширину окна связи и параметры модуляционного резонатора. Данное устройство предназначено для генерации импульсов мощного СВЧ-излучения и имеет следующий принцип работы. В цилиндрическом вакуумном диоде со взрывоэмиссионным катодом без внешнего фокусирующего магнитного поля формируется сплошной цилиндрический релятивистский электронный пучок с током, превышающим второй критический, который затем транспортируется через анодную сетку в двухзазорный резонатор в направлении, перпендикулярном к его наибольшей стенке. Зазоры резонатора представляют собой отрезки электродинамически связанных друг с другом прямоугольных волноводов. Электронный пучок попадает из первого зазора 7 во второй 8 , пролетая сквозь металлическую фольгу, которая располагается в стенке, разделяющей зазоры. Фактически первый зазор выполняет роль модулятора, реализующего воздействие обратной связи, а второй - камеры взаимодействия, предназначенной для формирования в ВК и последующего энергоотбора. При образовании ВК во втором зазоре резонатора возбуждается одна из его низших мод,

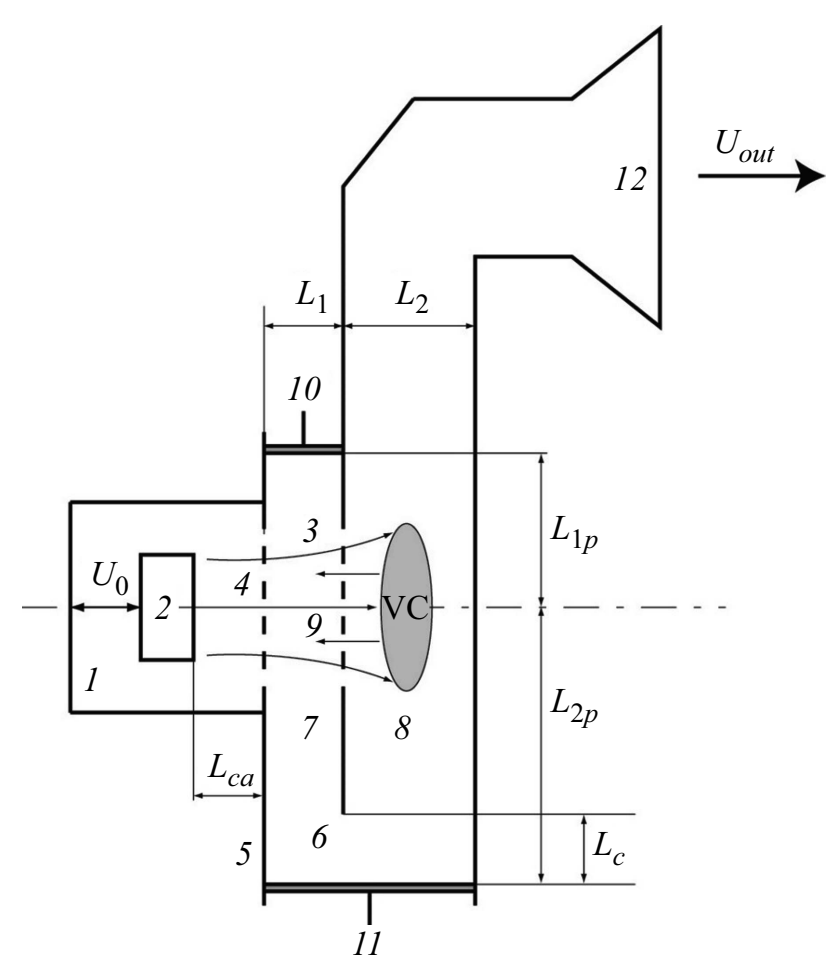

Рис. 1. Схема исследуемого виртода: 1 - электронная пушка, 2 - катод, 3 - сплошной релятивистский электронный поток, 4 - анодная сетка, 5 - двухзазорный резонатор, 6 - окно связи, 7 - первый зазор двухзазорного резонатора, 8 - второй зазор двухзазорного резонатора, $9-$ сетка в стенке между зазорами, 10 - плунжер, регулирующий высоту первого зазора, 11 - плунжер, регулирующий ширину окна связи, 12 - вывод мощности. Также на рисунке $U_{0}-$ ускоряющее напряжение, $L_{c a}-$ расстояние между катодом и анодом, $L_{1}-$ длина первой секции, $L_{2}-$ длина второй секции, $L_{1 p}$ - положение первого плунжера, $L_{2 p}$ - положение второго плунжера, $L_{c}-$ ширина окна связи, $U_{\text {out }}-$ сигнал, получаемый на выходе, ВК - схематическое обозначение области виртуального катода.

которая излучается в свободное пространство посредством рупорной антенны, подключенной через волновод к верхней границе второго зазора. Дополнительная электромагнитная обратная связь реализуется за счет электромагнитной волны, проникающей через окно обратной связи из второго зазора резонатора в первый, и позволяет увеличить КПД и стабильность частоты излучения виртода.

Заметим, что колебания электромагнитного поля во втором зазоре, создаваемые нестационарным ВК, возбуждают колебания поля в первом зазоре, что приводит к модуляции проходящего электронного потока на частоте осцилляций ВК. Вследствие этого важным фактором, определяющим геометрию резонатора, является соблюдение оптимального сдвига фаз между колебаниями высокочастотного поля на оси пучка в первом и втором зазорах. Согласно работе [29], в виртоде предпринята попытка создать режим синфазных полей: $\delta \phi=0$, где 
$\delta \phi-$ сдвиг фаз между полями в зазорах. Положения плунжеров 10, 11 выбираются таким образом, чтобы они отстояли на $\sim 3 l / 4$ от оси пучка, где $l-$ это длина волны колебаний выходного сигнала. В этом случае в первом зазоре образуется стоячая волна, состоящая из 3 полуволн и симметричная относительно оси пучка. Это приводит к тому, что пучок проходит через пучности поля в первом и втором зазорах и эффективно взаимодействует с электромагнитным полем. Очевидно, что частота колебаний выходного излучения полностью зависит от размеров резонатора и может изменяться при соответствующей механической подстройке плунжеров 10 и 11 (рис. 1).

\section{Исследование характеристик генерации}

Была проведена многопараметрическая численная оптимизация геометрии виртода с точки зрения повышения эффективности и стабильности частоты генерации. Определенные геометрические параметры системы соответствуют условиям и требованиям, предъявляемым к приборам подобного рода. Были выявлены следующие оптимальные геометрические параметры релятивистского виртода: радиус катода $r_{c}=43 \mathrm{~mm}$, радиус электронной пушки $r_{g}=47.3 \mathrm{~mm}$, расстояние между катодом и анодной сеткой $L_{c a}=24.4 \mathrm{~mm}$, длина первого зазора $L_{1}=23.7 \mathrm{~mm}$, расстояние между верхним поршнем в первом зазоре и осью пучка $L_{1 p}=166.5 \mathrm{~mm}$, расстояние между нижним поршнем и осью пучка $L_{2 p}=174.4 \mathrm{~mm}$, длина второго зазора $L_{2}=91.7 \mathrm{~mm}$, положение выходного волновода относительно оси пучка $L_{\text {out }}=272.4 \mathrm{~mm}$, ширина резонатора (одинаковая в обоих зазорах) $D=115.56 \mathrm{~mm}$, ширина окна связи $L_{b}=9.8 \mathrm{~mm}$.

Исследовано влияние параметров пучка на характеристики генерации виртода. Для этого была построена зависимость КПД от уровня питающего напряжения в пушечной части (рис. 2,a). Хорошо видно, что максимальный КПД виртода достигает величины порядка 4\% при ускоряющем напряжении $600 \mathrm{kV}$. При дальнейшем увеличении ускоряющего напряжения величина КПД генератора уменьшается, демонстрируя локальные максимумы и минимумы. При больших ускоряющих напряжениях $U_{0}>1.4 \mathrm{MV}$ КПД оказывается достаточно низким (менее $0.5 \%$ ). Оптимальному значению $U_{0}$ соответствует режим с наиболее стабильной частотой генерации. Отклонение уровня ускоряющего напряжения от оптимального в любую сторону приводит к ухудшению стабильности генерации, при этом увеличение $U_{0}$ ведет к задержке начала генерации. Аналогичные исследования были проведены при изменении величины тока РЭП (рис. 2,b). Зависимость КПД от тока оказывается более гладкой с одним характерным максимумом, соответствующим току порядка $15 \mathrm{kA}$.

Для исследования влияния шумового разброса электронов пучок инжектировался в систему с заданным раз-
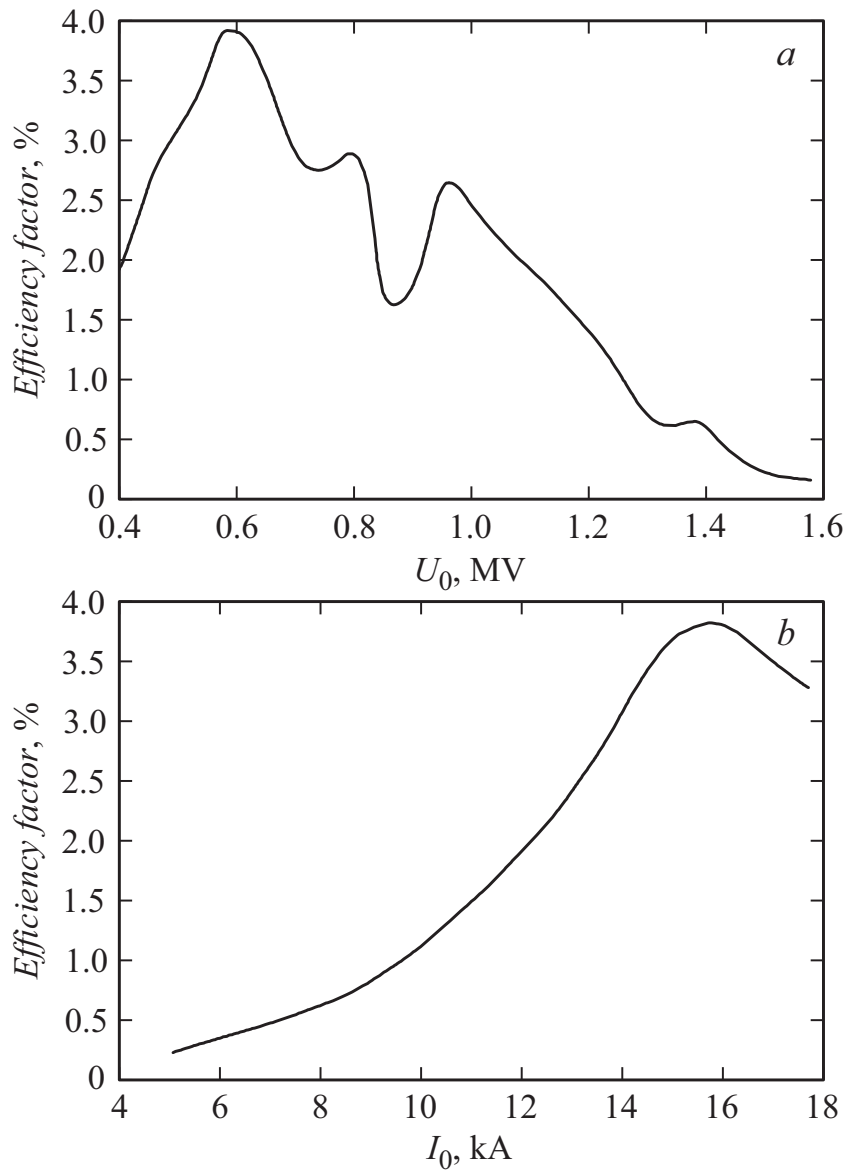

Рис. 2. Зависимости КПД виртода от ускоряющего напряжения $(a)$ и тока пучка $(b)$.

бросом по энергии и углам влета электронов в систему, при этом использовалась равномерная функция распределения. Таким образом, значения энергии инжектируемых электронов равномерно распределены в диапазоне $\left[E_{0}-\Delta E, E_{0}+\Delta E\right]$ (где $E_{0}-$ средняя начальная энергия пучка, $\Delta E$ - параметр разброса по энергии), а углы влёта относительно нормали к плоскости инжекции в диапазоне $[-\Delta \alpha, \Delta \alpha]$ (где $\Delta \alpha-$ параметр разброса по углам в градусах).

Обнаружено, что увеличение степени шумового разброса инжектируемых электронов ведет к уменьшению выходной мощности (рис. 3), при этом более существенное влияние оказывает разброс электронов по углам влета: так, при $\Delta \alpha>18$ и $\Delta E=0$ выходная мощность становится практически нулевой, и фактически происходит срыв генерации. Увеличение разброса по энергии $\Delta E$ от 0 до $75 \%$ (при $\Delta \alpha=0)$ ведет к падению выходной мощности в 3-4 раза. Также выявлена особенность, что величина $\Delta \alpha$, при которой происходит срыв генерации, уменьшается с ростом разброса по энергии в диапазоне $\Delta E$ от 0 до $5 \%$. При $\Delta E>5 \%$ данная величина, напротив, увеличивается с ростом $\Delta E$ (см. рис. 3 ). С физической точки зрения, срыв генерации в виртоде из-за 


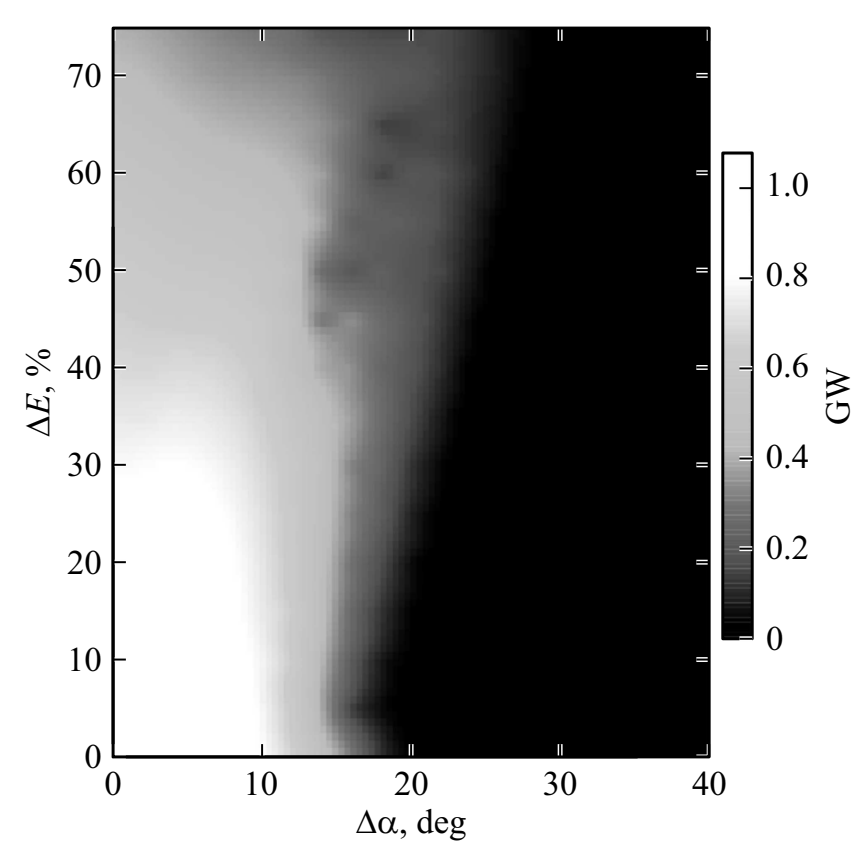

Рис. 3. Зависимость мощности выходного сигнала релятивистского виртода с шумовым разбросом инжектируемых электронов от параметров разброса по энергии $\Delta E$ и углам влета $\Delta \alpha$. Ток пучка $I_{0}=9 \mathrm{kA}, U_{0}=1 \mathrm{MV}$.

влияния шумового разброса обусловлен разрушением когерентной структуры виртуального катода.

Для исследования влияния модуляции инжектируемого электронного потока по скорости на характеристики генерации виртода на анодную сетку пушечной части прибора подавалось ускоряющее напряжение, представляющее собой сумму постоянной величины $U_{0}$ и синусоидальной составляющей $U_{0} A \sin (2 \pi f t)$, где $A$ - амплитуда модуляции, $f$ - частота модуляции. В результате электронный поток вылетает из пушечной части промодулированным по скорости.

Обнаружен резонансный характер зависимости интегральной выходной мощности виртода от частоты модуляции (рис. 4,a): мощность достигает максимального значения при совпадении $f$ с собственной частотой автономной генерации виртода, которая определяется частотой рабочей моды первого зазора двухзазорного резонатора. Таким образом, удается достигнуть существенного увеличения мощности и эффективности генерации вплоть до 13\%. Заметим, что введение дополнительной модуляции пучка также приводит к сокращению длительности переходного процесса и соответственно к более быстрому запуску генерации. Напомним, что сигнал на выходе автономного виртода появляется через некоторый промежуток времени: после переходного процесса, который может достигать $100 \mathrm{~ns}$.

Выходная мощность виртода быстро насыщается в зависимости от амплитуды модуляции $A$ (рис. 4,b), достигая близкого к максимальному значению при $A \sim 0.05$. Более того, в случае равенства $f$ собственной частоте виртода $(1.41 \mathrm{GHz}$ в рассматриваемом случае) зависимость $P(A)$ (сплошная кривая на рис. $4, b)$ демонстрирует постоянный рост, сменяющийся насыщением. В других случаях (например, при $f=1.3 \mathrm{GHz}$ ) зависимость выходной мощности от амплитуды модуляции (штриховая кривая на рис. $4, b$ ) более сложная и содержит участок спада, сменяющийся дальнейшим ростом. Такое поведение зависимости обусловлено нелинейным поведением амплитуд спектральных составляющих выходного сигнала, сумма которых определяет величину
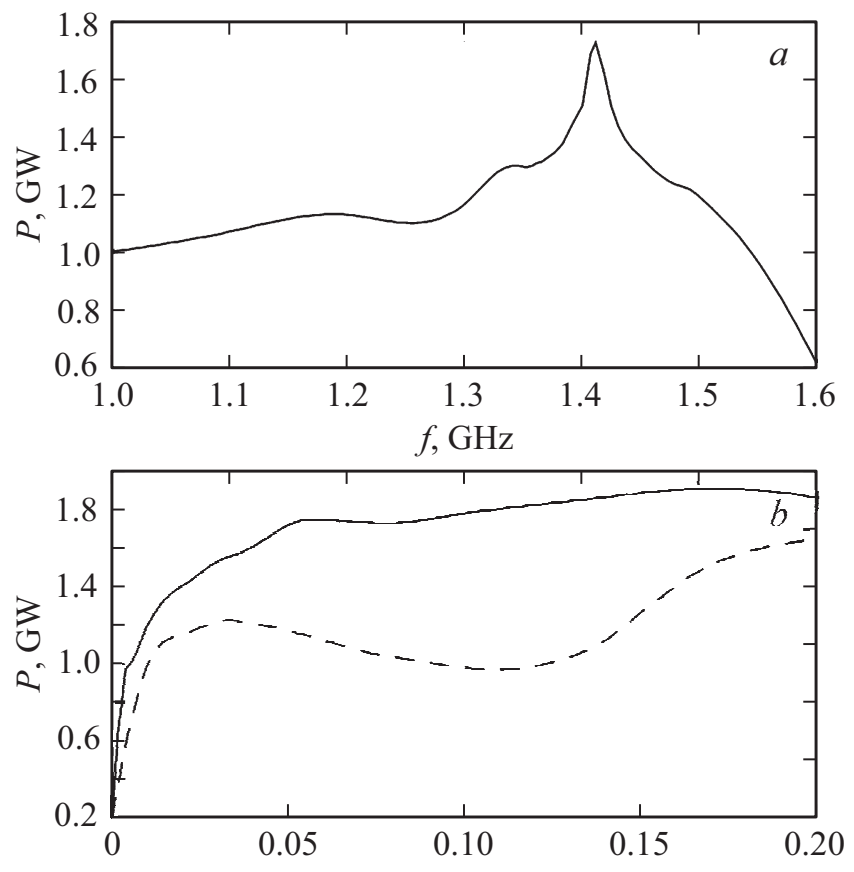

A

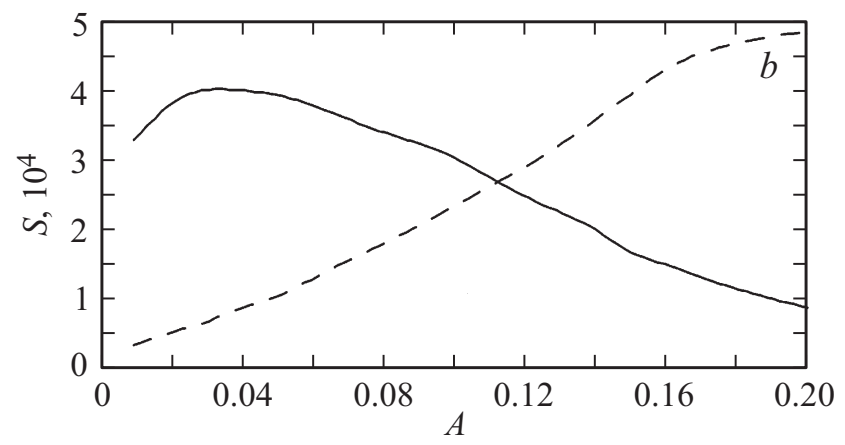

Рис. 4. $a-$ зависимость интегральной выходной мощности релятивистского виртода с модуляцией инжектируемого электронного потока по скорости от частоты модуляции $f$, амплитуда модуляции $A=5 \% . b-$ зависимость интегральной выходной мощности от амплитуды модуляции при частоте модуляции $f=1.41 \mathrm{GHz}$ (сплошная кривая), равной частоте генерации автономного виртода, и $f=1.3 \mathrm{GHz}$ (штриховая кривая). $c$ - зависимости амплитуд составляющих в спектре выходного сигнала на частоте автономной генерации виртода $1.41 \mathrm{GHz}$ (сплошная кривая) и частоте модуляции $1.3 \mathrm{GHz}$ (штриховая кривая) от амплитуды модуляции $A$; частота модуляции $f=1.3$. Т GHz от пучка $I_{0}=12.5 \mathrm{kA}, U_{0}=1 \mathrm{MV}$. Интервал времени, по которому проводилась оценка интегральной выходной мощности от 15 до $100 \mathrm{~ns}$. 
выходной мощности, при изменении $A$ (рис. 4,c). Действительно, если амплитуда составляющей на частоте модуляции демонстрирует постоянный рост с увеличением $A$, то амплитуда на собственной частоте сначала возрастает, а затем начинает уменышаться вследствие навязывания пучку модуляционной частоты, тем самым уменьшая эффективность взаимодействия пучка с модой, возбуждаемой в первом зазоре.

С физической точки зрения увеличение выходной мощности виртода при введении модуляции потока по скорости на частоте, близкой к собственной, обусловлено тем, что изменение скоростей, вновь подлетающих к области ВК-электронов, находится в фазе с его собственными колебаниями, и новые для ВК электроны имеют „правильные“ скорости, позволяющие сформировать более плотную структуру. Как следствие, выходная мощность генерации виртода увеличивается.

\section{Заключение}

В работе представлены результаты численного исследования характеристик генерации перспективной схемы генератора на виртуальном катоде с внешней обратной связью (виртода). Определены оптимальные геометрические параметры системы и параметры пучка. Исследовано влияние предмодуляции пучка по скорости и разброса электронов по скоростям и углам влета на характеристики генерации виртода. Показано, что введение дополнительной модуляции электронного потока на собственной частоте модуляционного резонатора приводит к резкому возрастанию эффективности генерации, которая в пике составляет $\sim 13 \%$. Это обусловлено эффектом резонансного взаимодействия пучка и электромагнитного поля. Также дополнительная модуляция приводит к сокращению длительности переходного процесса, что позволяет увеличить скорость выхода прибора в рабочий режим. Исследования влияния разброса электронов по скоростям и углам влета показали, что в широком диапазоне значений параметров разброса возможна эффективная генерация. Последнее говорит о низких требованиях, предъявляемых к качеству пучка, в виртоде. Так, разброс по скоростям электронов до $30 \%$ практически не оказывает влияния на характеристики генерации. Более того, при больших величинах разброса по скоростям генерация не срывается, хотя эффективность ее существенно снижается.

Полученные результаты позволяют считать генераторы на виртуальном катоде с дополнительной обратной связью перспективными для получения сверхмощного СВЧ-излучения. Они характеризуются высокой стабильностью частоты генерации и эффективностью для данного типа приборов.

Исследования выполнены при поддержке Министерства образования и науки РФ ( № 3.859.2017/4.6) и гранта президента РФ для молодых российских ученых кандидатов наук (МК-1163.2017.2).

\section{Список литературы}

[1] Benford J., Swegle J. A., Schamiloglu E. High Power Microwaves. CRC Press. Taylor and Francis Group. 2016.

[2] Collin R.E. Foundations for Microwave Engineering. John Wiley and Sons, Inc. 2001.

[3] Mahaffey R.A., Sprangle P.A., Golden J. et al. // Phys. Rev. Lett. 1977. Vol. 39. N 13. P. 843.

[4] Диденко А.Н., Красик Я.Е., Перелыгин С.Ф. и др. // Письма в ЖТФ. 1979. Т. 5. Вып. 6. С. 321.

[5] Sullivan D.J., Walsh J.E., Coutsias E.A. Virtual Cathode Oscillator (Vircator) Theory. Artech House Microwave Library. 1987.

[6] Granatstein V.L., Alexeeff I. High Power Microwave Sources. Artech House Microwave Library. 1987.

[7] Gold S.H., Nusinovich G.S. // Rev. Sci. Instruments. 1997. Vol. 68. N 11. P. 3945-3974.

[8] Дубинов А.Е., Селемир В.Д. // РЭ. 2002. Т. 47. № 6. С. 575.

[9] Singh G., Chaturvedi Shashank. // Phys. Plasmas. 2011. Vol. 18. P. 063104.

[10] Li L., Liu L., Cheng G. et al. // J. Appl. Phys. 2009. Vol. 105. N 12. P. 123301.

[11] Li L., Cheng G., Zhang L. et al. // J. Appl. Phys. 2011. Vol. 109. P. 074504.

[12] Бадарин А.А., Куркин С.А., Короновский А.А. и др. // Письма в ЖТФ. 2015. Т. 41. Вып. 23. С. 72-80.

[13] Hramov A.E., Kurkin S.A., Koronovskii A.A. et al. // Phys. Plasmas. 2012. Vol. 19. N 11. P. 112101.

[14] Ju J., Cai D., Du G. et al. // IEEE Transactions on Plasma Science. 2015. Vol. 43. P. 3522-3526.

[15] Бадарин А.А., Куркин С.А., Храмов А.Е. // Известия РАН. Серия физическая. 2015. Т. 79. № 12. С. 1646-1649.

[16] Бадарин А.А., Куркин С.А., Короновский А.А. и др. // Физика плазмы. 2017. Т. 43. № 3. С. 284-292.

[17] Fazio M.V., Kinross-Wright J., Haynes B. et al. // Appl. Phys. 1989. Vol. 66. P. 2675-2677.

[18] Hramov A.E., Koronovskii A.A., Kurkin S.A. // Phys. Lett. A. 2010. Vol. 374. P. 3057-3066.

[19] Куркин С.А., Короновский А.А., Храмов А.Е. // Письма в ЖТФ. 2011. Т. 37. Вып. 8. С. 26-33.

[20] Phrolov N.S., Koronovskii A.A., Kalinin Y. et al. // Phys. Lett. A. 2014. Vol. 378. P. 2423-2428.

[21] Burkhart S.C., Scarpetty R.D., Lundberg R.L. // J. Appl. Phys. 1985. Vol. 58. N 28.

[22] Hoeberling R.F., Fazio M.V. // IEEE Trans. Electromagnetic Compatibility. 1992. Vol. 34. P. 252-258.

[23] Abubakirov E.B., Konyushkov A.P. // IEEE Transactions on Plasma Science 2010. Vol. 38. P. 1285-1291.

[24] Gadetskii N.N., Magda I.I., Naisteter S.I. et al. // Plasma Phys. Rep. 1993. Vol 19. P. 273.

[25] Shlapakovski A.S., Queller T., Bliokh Y. et al. // IEEE Transactions on Plasma Sci. 2012. Vol. 40. N 6. P. 1607-1617.

[26] Kovalchuk B.M., Polevin S.D., Tsygankov R.V. et al. // IEEE Transactions on Plasma Sci. 2010. Vol. 38. P. 2819-2824.

[27] Kitsanov S.A., Klimov A.I., Korovin S.D. et al. // IEEE Trans. Plasma Sci. 2002. Vol. 30. P. 274-285.

[28] Birdsall C.K., Langdon A.B. // Plasma Phys. Via Computer Simulation. CRC press. 2004.

[29] Кицанов С.А., Климов А.И., Коровин С.Д. и др. // ЖТФ. 2002. Т. 72. Вып. 5. С. 82-90. 